\title{
Wide Spectral Photoresponse of Layered Platinum Diselenide-Based Photodiodes
}

\author{
Chanyoung Yim, ${ }^{\dagger, \ddagger}$ Niall McEvoy, ${ }^{\S, \|}$ Sarah Riazimehr, ${ }^{\dagger, \perp}$ Daniel S. Schneider, ${ }^{\dagger}$ Farzan Gity, ${ }^{\#}$
}

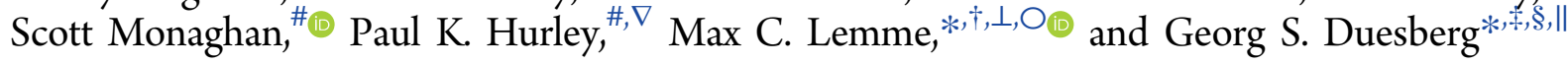

${ }^{\dagger}$ Department of Electrical Engineering and Computer Science, University of Siegen, Hölderlinstraße 3, 57076 Siegen, Germany

${ }^{\ddagger}$ Institute of Physics, EIT 2, Faculty of Electrical Engineering and Information Technology, Universität der Bundeswehr München, Werner-Heisenberg-Weg 39, 85577 Neubiberg, Germany

${ }^{\S}$ School of Chemistry, Trinity College Dublin, Dublin 2, Ireland

${ }$ Centre for the Research on Adaptive Nanostructures and Nanodevices (CRANN) and Advanced Materials and BioEngineering Research (AMBER), Trinity College Dublin, Dublin 2, Ireland

${ }^{\perp}$ Chair of Electronic Devices, Faculty of Electrical Engineering and Information Technology, RWTH Aachen University, Otto-Blumenthal-Str. 2, 52074 Aachen, Germany

${ }^{\#}$ Tyndall National Institute, University College Cork, Lee Maltings, Dyke Parade, Cork T12 R5CP, Ireland

${ }^{\nabla}$ Department of Chemistry, University College Cork, Lee Maltings, Dyke Parade, Cork T12 R5CP, Ireland

$\mathrm{O}_{\mathrm{AMO}} \mathrm{GmbH}$, Advanced Microelectronic Center Aachen, Otto-Blumenthal-Str. 25, 52074 Aachen, Germany

\section{Supporting Information}

ABSTRACT: Platinum diselenide $\left(\mathrm{PtSe}_{2}\right)$ is a group-10 transition metal dichalcogenide (TMD) that has unique electronic properties, in particular a semimetal-to-semiconductor transition when going from bulk to monolayer form. We report on vertical hybrid Schottky barrier diodes (SBDs) of two-dimensional (2D) $\mathrm{PtSe}_{2}$ thin films on crystalline n-type silicon. The diodes have been fabricated by transferring large-scale layered $\mathrm{PtSe}_{2}$ films, synthesized by thermally assisted conversion of predeposited $\mathrm{Pt}$ films at back-end-of-the-line CMOS compatible temperatures, onto $\mathrm{SiO}_{2} / \mathrm{Si}$ substrates. The
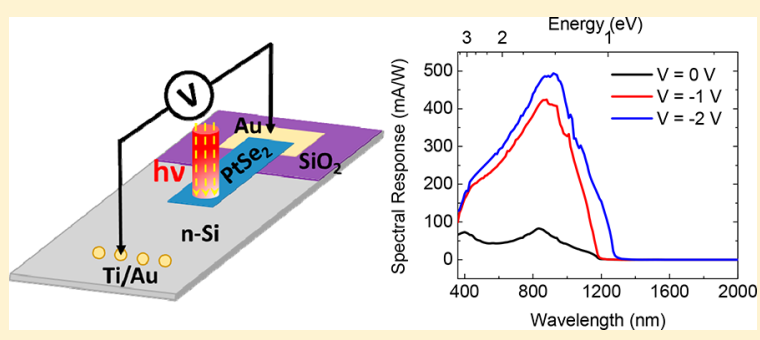
diodes exhibit obvious rectifying behavior with a photoresponse under illumination. Spectral response analysis reveals a maximum responsivity of $490 \mathrm{~mA} / \mathrm{W}$ at photon energies above the Si bandgap and relatively weak responsivity, in the range of 0.1-1.5 mA/W, at photon energies below the Si bandgap. In particular, the photoresponsivity of $\mathrm{PtSe}_{2}$ in infrared allows $\mathrm{PtSe}{ }_{2}$ to be utilized as an absorber of infrared light with tunable sensitivity. The results of our study indicate that $\mathrm{PtSe}_{2}$ is a promising option for the development of infrared absorbers and detectors for optoelectronics applications with low-temperature processing conditions.

KEYWORDS: Two-dimensional transition metal dichalcogenide, platinum diselenide, Schottky barrier diode, photodetector, spectral response, IR photodiode

$\mathrm{T}$ wo-dimensional (2D) layered transition metal dichalcogenides (TMDs) are under investigation for use in future (opto)electronic device applications owing to their nanoscale thickness as well as their unique electrical and optical properties. $^{1-3}$ To date, most studies on TMD-based electronic devices have focused on group-6 TMDs such as molybdenum/ tungsten $(\mathrm{Mo} / \mathrm{W})$ disulfide/diselenide $\left(\mathrm{S}_{2} / \mathrm{Se}_{2}\right){ }^{4-9}$ Various scalable and controllable growth techniques for such materials have been developed, mostly based on chemical vapor deposition (CVD) or vapor phase deposition, ${ }^{10-16}$ and the opportunity for manufacturable device applications has become much broader, leading to increased research on transistors, sensors, and optoelectronic devices. ${ }^{9,17-21}$

In contrast to group-6 TMDs, group-10 TMDs, such as platinum/palladium $(\mathrm{Pt} / \mathrm{Pd})$ disulfide/diselenide $\left(\mathrm{S}_{2} / \mathrm{Se}_{2}\right)$, have thus far been little explored. Even though theoretical studies on the electronic properties of group-10 TMDs started in the $1960 s^{22-24}$ experimental studies on their use for electronic device applications have only been introduced very recently. ${ }^{25-28}$ In particular, $\mathrm{PtSe}_{2}$ has drawn attention due to its unique electronic property whereby theoretical studies proposed a transition from semimetal to semiconductor as the layer number decreases. ${ }^{24,29,30}$ Wang et al. were able to grow $\mathrm{PtSe}_{2}$ by direct selenization of $\mathrm{Pt}$ single crystals under ultrahigh vacuum conditions and experimentally demonstrated that monolayer $\mathrm{PtSe}_{2}$ is semiconducting with an indirect

Received: November 27, 2017

Revised: January 24, 2018

Published: February 20, 2018 
(a)

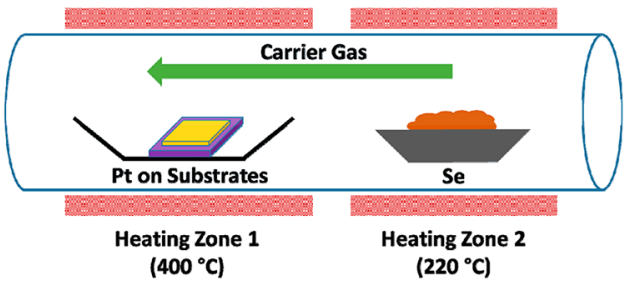

(c)

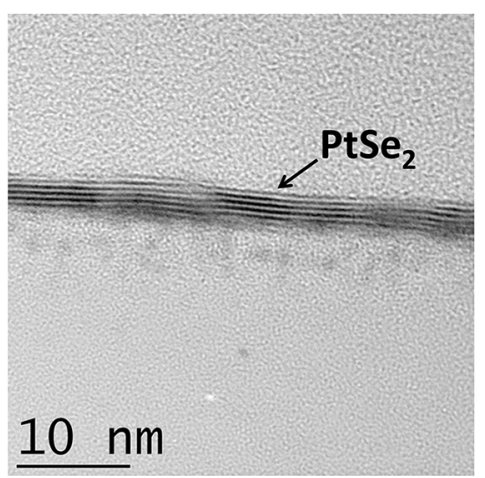

(b)

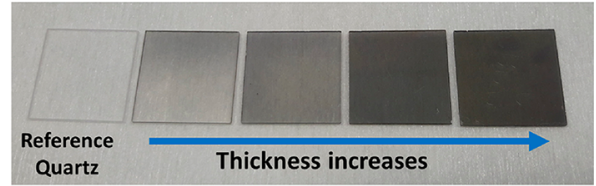

(d)

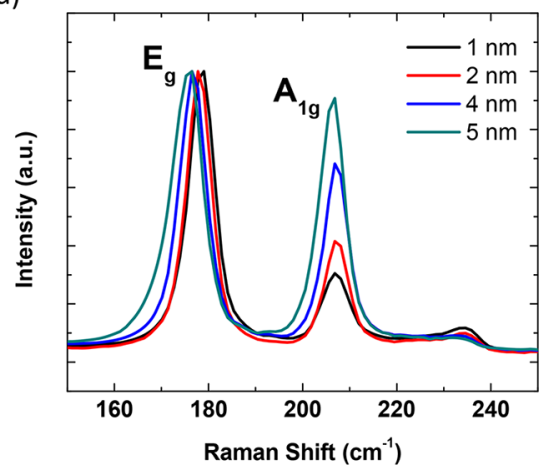

Figure 1. (a) Schematic diagram of the $\mathrm{PtSe}_{2}$ film synthesis process and (b) photograph of the $\mathrm{PtSe}_{2}$ samples (bottom) synthesized on quartz substrates $(2 \mathrm{~cm} \times 2 \mathrm{~cm})$ with increasing initial Pt deposition thicknesses $(1,2,3$, and $5 \mathrm{~nm})$ from left to right. (c) Cross-sectional TEM image of the $\mathrm{PtSe}_{2}$ selenized from a $1 \mathrm{~nm}$ thick Pt layer. Note: in this case the $\mathrm{PtSe}_{2}$ was formed on $85 \mathrm{~nm}$ amorphous $\mathrm{SiO}_{2}$ thermally grown on silicon. (d) Raman spectra of $\mathrm{PtSe}_{2}$ films selenized from different Pt thicknesses $\left(1,2,4\right.$, and $5 \mathrm{~nm}$ ) normalized to the intensity of the $\mathrm{E}_{\mathrm{g}}$ mode.

bandgap of $1.2 \mathrm{eV}$, unlike semimetallic bulk $\mathrm{PtSe}_{2}$ with zero bandgap. ${ }^{31}$ Furthermore, with recent developments in the synthesis of layered $\mathrm{PtSe}_{2}$ thin films in a scalable and controllable way, it has been utilized in various nanoelectronic devices, including gas sensors, photodetectors, and photovoltaic cells, showing notable performance. ${ }^{25,30}$ Due to this thicknessdependent transition, a wide range of device applications, especially in optoelectronics, can be envisioned for $\mathrm{PtSe}_{2}$. In particular, the thickness dependence of the bandgap in $\mathrm{PtSe}_{2}$ suggests the possibility of infrared (IR) photodetection. Despite the fact that various 2D TMDs have been employed in photodetectors, ${ }^{32}$ IR detection has rarely been achieved as most TMDs are semiconducting with an appreciable bandgap.

Vertically illuminated Schottky photodetectors can be fabricated by forming simple metal-semiconductor contacts. They are advantageous for high-speed applications due to their majority-carrier-dominant current-flow mechanism, and Schottky photodetectors based on III-V materials (e.g., GaAs, InGaAs, InAlAs, etc.) have also been utilized for nearIR/IR detection. ${ }^{33,34}$ However, the high reflectance of conventional metallic Schottky contact layers deteriorates the device performance, resulting in low efficiency. Though transparent conductors, such as indium tin oxide (ITO), were introduced to overcome this drawback, ${ }^{35,36}$ it is necessary to use additional layers for antireflectance and enhancement of light absorption. Compared to transparent-conductor-based IR photodetectors, 2D-material-based photodetectors can be thinned down relatively easily by reducing the thickness of the $2 \mathrm{D}$ top contact layers to a few nanometers as the $2 \mathrm{D}$ layer acts as a conductive electrode as well as a light absorber. In addition, it is possible to tune the optical properties of the $2 \mathrm{D}$ layers by controlling the layer thickness, enabling a broad range of wavelength detection. The mechanical flexibility of layered materials also strengthens the case for their future use in flexible device applications. Graphene has been studied as an active layer for graphene-Si Schottky photodiodes. ${ }^{37,38}$ Its gapless semimetallic nature allows graphene to be utilized as an IR detector; ${ }^{38}$ however, the limited light absorption of graphene, owing to its monolayer structure, and relatively complex processes associated with stacking multilayers hinder broader applications of graphene-based IR detection. On the contrary, multilayer $\mathrm{PtSe}_{2}$, with a small $(<\sim 0.2 \mathrm{eV})$ or zero bandgap, ${ }^{31}$ can be expected to absorb light over a very wide energy spectrum and generate photon-induced charge carriers, thus enabling broadband photodetection.

In this work, we investigate the wide spectral photoresponse of $\mathrm{PtSe}_{2}$-based electronic devices. In our previous study, we demonstrated $\mathrm{PtSe}_{2}$-based photodiodes and examined their general photoconductivity and photovoltaic effects under white light illumination. ${ }^{25}$ The layered $\mathrm{PtSe}_{2}$ thin films were fabricated in a similar manner with various thicknesses synthesized by thermally assisted conversion (TAC) of predeposited Pt layers. Schottky barrier diodes (SBDs) were fabricated by transferring layered $\mathrm{PtSe}_{2}$ thin films onto n-type $\mathrm{Si}$ $(\mathrm{n}-\mathrm{Si})$. Here, in addition to the basic investigation of the electrical characteristics of the hybrid $\mathrm{PtSe}_{2} / \mathrm{n}-\mathrm{Si} \mathrm{SBDs}$ by dc current-voltage measurements in ref 25 , the photoresponse of the SBDs was comprehensively examined over a wavelength range of $360-2000 \mathrm{~nm}(0.62-3.44 \mathrm{eV})$. It was found that there is a notable difference in responsivity depending on the photon energy range. A responsivity of $490 \mathrm{~mA} / \mathrm{W}$ and a relatively weak responsivity of $0.1-1.5 \mathrm{~mA} / \mathrm{W}$ were obtained at photon energies above and below the $\mathrm{Si}$ bandgap, respectively. In particular, our current study enables deeper understanding and quantification of the photoresponse of the $\mathrm{PtSe}_{2}$-based photodetectors by offering insight into their spectral response and photoconductivity. The results show that $\mathrm{PtSe}_{2}$ is a 
(a)

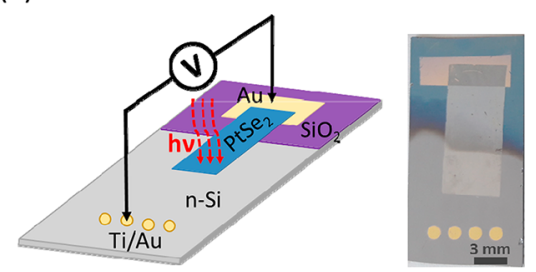

(c)

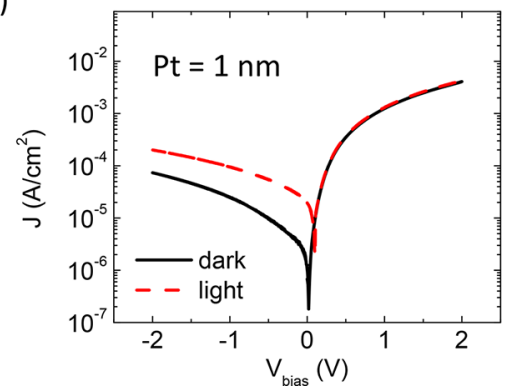

(b)

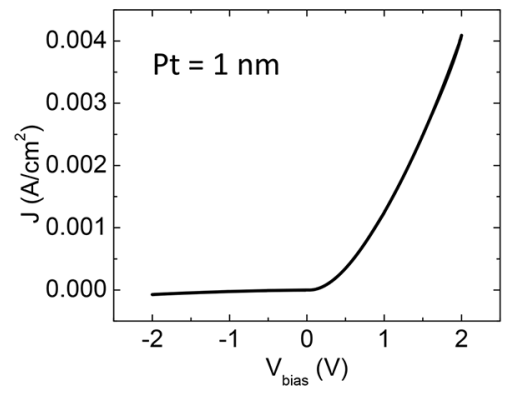

(d)

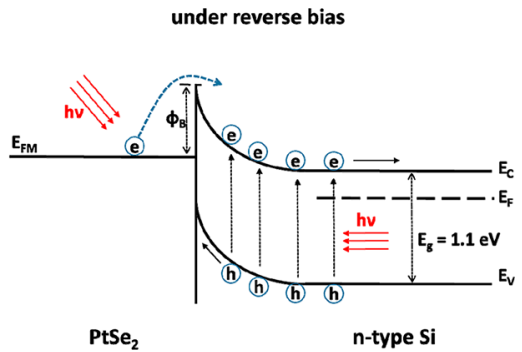

Figure 2. (a) Schematic diagram (left) and photograph (right) of a PtSe $2 / n-S i S B D$. dc $J-V$ plots of the SBD with a PtSe film selenized from $1 \mathrm{~nm}$ thick Pt (b) in dark conditions on a linear scale, and (c) in dark and under illumination with a white-light source $\left(P_{\text {in }}=12.3 \mathrm{~mW} / \mathrm{cm}^{2}\right)$ on a semilogarithmic scale. (d) Energy band diagram of the $\mathrm{PtSe}_{2} / \mathrm{n}-\mathrm{Si}$ Schottky junction under reverse bias. $\varphi_{\mathrm{B}}$ is the Schottky barrier height, and $E_{\mathrm{FM}}$ denotes the Fermi energy level of $\mathrm{PtSe}_{2} . E_{\mathrm{C}}, E_{\mathrm{F}}, E_{\mathrm{V}}$, and $h \nu$ are the conduction band edge, Fermi energy level, valence band edge of $\mathrm{n}-\mathrm{Si}$, and the incident photon energy, respectively.

promising candidate for the development of infrared absorbers and detectors.

A schematic of the furnace for the TAC process is shown in Figure 1a. The thicknesses of the $\mathrm{PtSe}_{2}$ films were controlled by depositing initial Pt layers with different thicknesses. A photograph of $\mathrm{PtSe}_{2}$ films with 1, 2, 3, and $5 \mathrm{~nm}$ of starting Pt grown on quartz substrates is shown in Figure 1b. It has been revealed that the initial $\mathrm{Pt}$ thickness expands approximately four times after selenization. ${ }^{39}$ As the single layer $\mathrm{PtSe}_{2}$ thickness is $\sim 0.7 \mathrm{~nm},{ }^{27,40}$ the number of layers for the $\mathrm{PtSe}_{2}$ films selenized from 1, 2, 3, and $5 \mathrm{~nm}$ thick initial Pt layers can be estimated as approximately 6, 11, 17, and 29 layers, respectively. It should be stressed that a relatively low growth temperature (of $400{ }^{\circ} \mathrm{C}$ ) is used in this process. This is an advantage over other TMD growth conditions based on chemical vapor deposition (CVD) or vapor phase deposition, ${ }^{14-16}$ wherein higher process temperatures $\left(>600{ }^{\circ} \mathrm{C}\right)$ are necessary for large-scale growth. This suggests that generally $\mathrm{PtSe}_{2}$ can be used in standard back-end-of-line (BEOL) processing of classic semiconductor technology for cointegration of electronic, optoelectronic, or nanoelectromechanical system (NEMS) applications. ${ }^{41-43}$

A cross-sectional transmission electron microscopy (TEM) image of the $\mathrm{PtSe}_{2}$ synthesized from the $1 \mathrm{~nm}$ thick Pt initial layer (Figure 1c) shows a clear multilayered structure with 5-6 layers of $\mathrm{PtSe}_{2}(\sim 3.5 \mathrm{~nm}$ thick). This shows that the initial metal layer is converted entirely to layered $\mathrm{PtSe}_{2}$ by the TAC process. It also can be seen that the layers are well aligned parallel to the substrate, even though it is known that TACderived films are polycrystalline. $9,25,30$

Hall measurements were performed for a $\mathrm{PtSe}_{2}$ film with the same thickness at room temperature in order to investigate the electronic properties of $\mathrm{PtSe}_{2}$. Both $\mathrm{DC}$ and $\mathrm{AC}$ magnetic field Hall-effect measurements indicate that the $\mathrm{PtSe}_{2}$ film yields net $p$-type carriers with a concentration of $1.89 \times 10^{20} \mathrm{~cm}^{-3}$ and Hall hole mobility of $3.5 \mathrm{~cm}^{2} \mathrm{~V}^{-1} \mathrm{~s}^{-1}$, resulting in a resistivity of $9.5 \times 10^{-3} \Omega \cdot \mathrm{cm}$. The relatively low Hall mobility value, compared to Hall mobility values of other TMDs, ${ }^{44,45}$ can be attributed to the high free carrier concentration and to its polycrystalline structure with randomly distributed grains of the TAC-grown $\mathrm{PtSe}_{2} \cdot{ }^{25,30}$ More details on the Hall measurements can be found in the Supporting Information (Figure S1 and Table S1). Raman spectra of $\mathrm{PtSe}_{2}$ films on $\mathrm{SiO}_{2} / \mathrm{Si}$ substrates with different thicknesses, synthesized from initial $\mathrm{Pt}$ thicknesses of 1, 2, 4, and $5 \mathrm{~nm}$, are depicted in Figure $1 \mathrm{~d}$. Two dominant peaks are observed at $\sim 177$ and $\sim 206 \mathrm{~cm}^{-1}$, which represent the $\mathrm{E}_{\mathrm{g}}$ and $\mathrm{A}_{\mathrm{lg}}$ Raman active modes of layered $\mathrm{PtSe}_{2}$ with a 1T-type crystal structure, respectively. The $\mathrm{E}_{\mathrm{g}}$ mode is related to an in-plane vibration of Se atoms, and the $\mathrm{A}_{1 \mathrm{~g}}$ mode reflects an out-of-plane vibration of Se atoms. A higher relative $A_{1 g}$ peak intensity, in combination with a slight red shift of the $E_{g}$ peaks, is observed as the films get thicker. As presented in a previous report, ${ }^{30}$ the rise in intensity of the $A_{1 g}$ mode can be attributed to an increased out-of-plane contribution, which may stem from increased van der Waals interactions in the thicker films.

As-grown $\mathrm{PtSe}_{2}$ films with various thicknesses were transferred onto prepatterned $\mathrm{n}-\mathrm{Si}$ substrates using a polymersupported transfer method, resulting in $\mathrm{PtSe}_{2} / \mathrm{n}-\mathrm{Si} \mathrm{SBDs}$. A schematic diagram and photograph of the fabricated device are shown in Figure 2a, and details of the fabrication process are described in the Methods section. Initially, electrical measurements of the devices were carried out in dark conditions. Figure $2 \mathrm{~b}$ depicts the dc current density-voltage $(J-V)$ plots of the device with a $\mathrm{PtSe}_{2}$ film grown from a $1 \mathrm{~nm}$ thick Pt layer. In dark conditions, clear rectifying behavior was observed, indicating that the device acts as a SBD. The current-voltage characteristics of SBDs follow thermionic emission theory and 
(a)

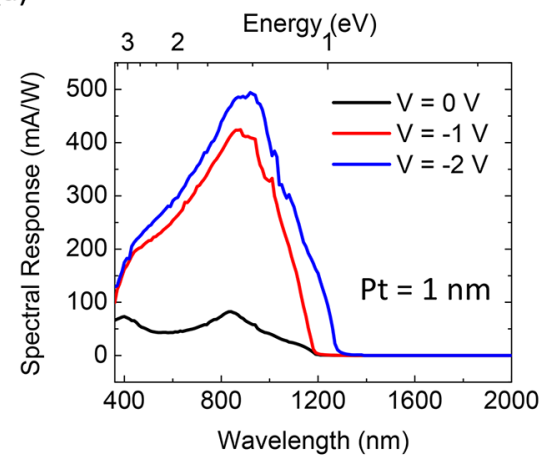

(c)

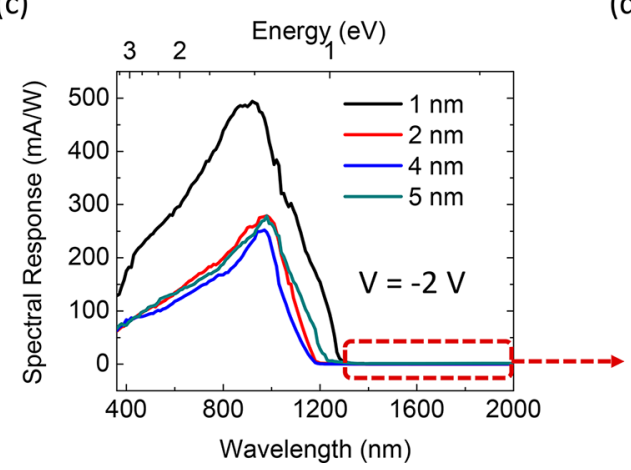

(b)

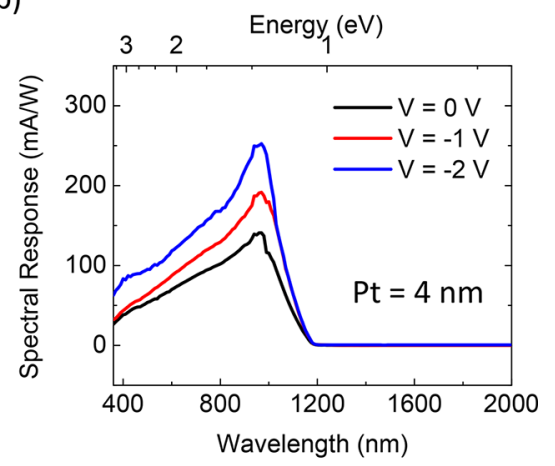

(d)

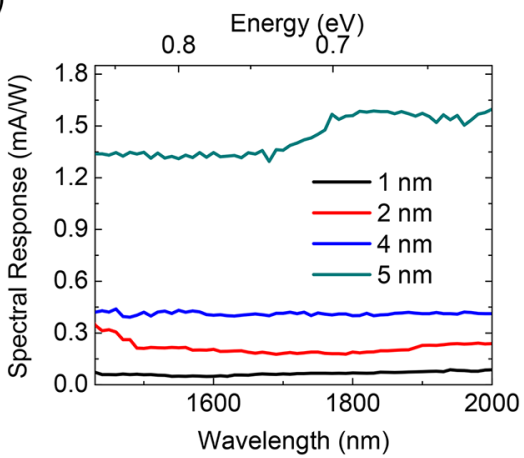

Figure 3. Absolute SR plots of the PtSe $e_{2} / \mathrm{n}-\mathrm{Si} \mathrm{SBDs}$ with $\mathrm{PtSe}_{2}$ films grown from (a) $1 \mathrm{~nm}$ and (b) $4 \mathrm{~nm}$ thick Pt layers under various reverse dc biases, wherein the light power density varies from 1 to $55 \mu \mathrm{W} \mathrm{cm}$-2 over the spectral range. (c) Absolute SR plot of SBDs with various PtSe ${ }_{2}$ thicknesses under a dc bias of $-2 \mathrm{~V}$ and $(\mathrm{d})$ enlarged SR plots as in $(\mathrm{c})$ in the infrared region.

their performance can be estimated by extracting typical diode parameters, including the ideality factor $(n)$, Schottky barrier height $\left(\varphi_{\mathrm{B}}\right)$, and series resistance $\left(R_{\mathrm{S}}\right) \cdot{ }^{46,47}$ Based on Cheung's method, ${ }^{48}$ the diode parameters of the device were determined from the dc $J-V$ measurement data, resulting in $n=1.7, \varphi_{\mathrm{B}}=$ $0.71 \mathrm{eV}$, and $R_{\mathrm{S}}=1.5 \mathrm{k} \Omega$. As a rule of thumb, for practical applications the ideality factor of a diode should be less than two $(1<n<2)$. Note that the ideality factor of the device is within this range, despite no optimization of the contacts or interfaces. Thus, this simple device-fabrication scheme is able to produce realistic diodes, which could be further improved by optimization of the fabrication process, such as development of a defect-free film transfer technique and implementation of guard rings.

The $\mathrm{PtSe}_{2}$ region of the device was illuminated with a whitelight source $\left(P_{\text {in }}=12.3 \mathrm{~mW} / \mathrm{cm}^{2}\right)$ to examine its general photoresponse. In this way, the light was able to transmit through the thin $\mathrm{PtSe}_{2}$ films and also interact with underlying $\mathrm{n}$-Si bulk. An obvious photoconductivity effect was observed from the $J-V$ plots of the $\mathrm{PtSe}_{2} / \mathrm{n}-\mathrm{Si} \mathrm{SBD}$, as presented in Figure 2c. While the SBD has a very low off-current under reverse bias in the dark, it shows a notable increase of the current under illumination. This is because the incident photons induce additional charge carriers, which contribute to a photocurrent. Based on the extracted diode parameters, an energy band diagram of the $\mathrm{PtSe}_{2} / \mathrm{n}$-Si Schottky junction under reverse bias can be derived (Figure 2d). The photon-induced charge carriers can be generated in the $\mathrm{PtSe}_{2}$ layer, in the depletion layer, and the silicon, depending on the incident photon energy and the absorption coefficients. When the incident photon energy $(h \nu)$ is $\varphi_{\mathrm{B}}<h \nu<E_{\mathrm{g}}$, where $E_{\mathrm{g}}$ is the bandgap of $\mathrm{Si}(\sim 1.1 \mathrm{eV})$, only electrons excited in the $\mathrm{PtSe}_{2}$ can contribute to the photocurrent. In the case of $E_{\mathrm{g}} \leq h \nu$, electron-hole pairs generated at the depletion region of the $\mathrm{PtSe}_{2}-\mathrm{Si}$ junction as well as the $\mathrm{Si}$ region can also contribute to the photocurrent. Similar white-light photoconductivity effects were observed for other $\mathrm{PtSe}_{2} / \mathrm{n}$-Si SBDs with different $\mathrm{PtSe}_{2}$ film thicknesses. The $J-V$ plots of diodes with $\mathrm{PtSe}_{2}$ derived from 2, 4, and $5 \mathrm{~nm}$ thick Pt layers are presented in Figure S2 of the Supporting Information.

Spectral response (SR) measurements of the $\mathrm{PtSe}_{2} / \mathrm{n}-\mathrm{Si}$ SBDs were carried out in order to examine how photons of different wavelength (energy) contribute to the generation of photocurrent. The absolute $\mathrm{SR}$ of the $\mathrm{PtSe}_{2} / \mathrm{n}-\mathrm{Si} \mathrm{SBDs}$ was measured over a broad wavelength range (360-2000 nm, $0.62-3.44 \mathrm{eV}$ ) under zero and reverse dc biases. The SR data of the SBDs with $\mathrm{PtSe}_{2}$ films grown from 1 and $4 \mathrm{~nm}$ starting $\mathrm{Pt}$ are plotted in Figure $3 \mathrm{a}, \mathrm{b}$, respectively. Higher responsivity is observed at a higher reverse bias for both devices. They exhibit very similar behavior showing maximum SR peaks at $\sim 970 \mathrm{~nm}$ $(\sim 1.28 \mathrm{eV})$, which is the fingerprint of absorption in the bottom $\mathrm{n}$-Si sublayer. Very low responsivity is observed at energies below the Si bandgap $\left(E_{\mathrm{g}}<\sim 1.1 \mathrm{eV}\right)$, implying that $\mathrm{PtSe}_{2}$ solely contributes to the $\mathrm{SR}$ in this region. Additional SR data of the SBDs with $\mathrm{PtSe}_{2}$ films from 2 and $5 \mathrm{~nm}$ starting $\mathrm{Pt}$ are presented in Figure $\mathrm{S} 4$ of the Supporting Information. The $\mathrm{SR}$ of the SBDs with different $\mathrm{PtSe}_{2}$ thicknesses under dc bias of $-2 \mathrm{~V}$ are compared in Figure $3 \mathrm{c}$ and zoomed in on the IR response in Figure 3d. All devices are responsive from the ultraviolet (UV) to the near-infrared (NIR) region. The SBD with a $\mathrm{PtSe}_{2}$ layer from $1 \mathrm{~nm}$ starting $\mathrm{Pt}$ shows a high maximum responsivity of $\sim 490 \mathrm{~mA} / \mathrm{W}$, while the three devices with thicker $\mathrm{PtSe}_{2}$ show lower maximum responsivities of 250-270 $\mathrm{mA} / \mathrm{W}$. These values are in the range of the responsivity of recently reported graphene/Si heterojunction photodetectors $(270-730 \mathrm{~mA} / \mathrm{W}),^{37,38,49}$ and higher than that of multilayer 
(a)

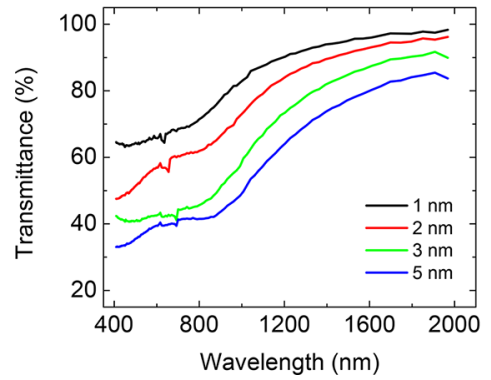

(b)

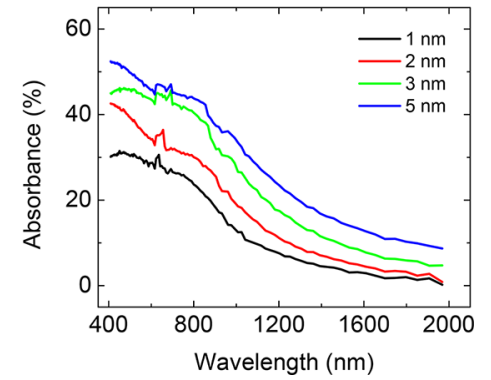

Figure 4. (a) Transmittance and (b) absorbance of the $\mathrm{PtSe}_{2}$ thin films with various thicknesses, selenized from 1, 2, 3, and $5 \mathrm{~nm}$ thick initial Pt layers on quartz substrates.

$\mathrm{MoS}_{2}$-based photodetectors $(10-210 \mathrm{~mA} / \mathrm{W}) .^{9,50,51}$ However, the responsivity begins to drop dramatically in the IR region due to the exclusion of the Si contribution to the photocurrent, showing a responsivity range of $0.1-1.5 \mathrm{~mA} / \mathrm{W}$ with a clear $\mathrm{PtSe}_{2}$ thickness dependence. This is consistent with previous graphene/Si photodetectors, ${ }^{38}$ which give a responsivity of less than $0.2 \mathrm{~mA} / \mathrm{W}$ in the IR region. ${ }^{38}$ Such a weak responsivity in the IR region can be explained by the internal photoemission effect (IPE), whereby electrical carriers generated in the (semi)metallic layer by photon absorption overcome the Schottky barrier and contribute to the photocurrent. ${ }^{52}$ Unlike typical IPE-based Schottky photodetectors, the $\mathrm{PtSe}_{2} / \mathrm{n}-\mathrm{Si}$ SBDs do not show any noticeable drop in responsivity at the energy of their Schottky barrier height $(0.7 \mathrm{eV}, 1770 \mathrm{~nm})$. The additional photocurrent energies below the Schottky barrier height could be caused by the contribution of defect-induced traps at the interface, considering the $\mathrm{PtSe}_{2}$ film transfer processes wherein wet-etching processes of $\mathrm{SiO}_{2}$ layers are included. Furthermore, the additional photocurrent could stem from the contribution of randomly distributed semiconducting few-layered $\mathrm{PtSe}_{2}$ patches. As previously reported, ${ }^{30}$ TACgrown $\mathrm{PtSe}_{2}$ has a polycrystalline structure with randomly distributed grains, consisting of multilayer $\mathrm{PtSe}_{2}$ with several different layer numbers. Similar structures have also been observed for TAC-grown $\mathrm{MoS}_{2}{ }^{53}$ The incident photons with lower energies than the Schottky barrier height can induce electron-hole pairs at the partially distributed semiconducting few-layered $\mathrm{PtSe}_{2}$ crystallites with small bandgap $(<0.3 \mathrm{eV})$, contributing additional photocurrent. Silicon Schottky photodiodes based on IPE have been frequently used for IR detection owing to their simple structure and ease of fabrication. ${ }^{54-56}$ The IR responsivity of the $\mathrm{PtSe}_{2}$-based Schottky diodes is comparable to the responsivity of conventional IPE-based Schottky photodetectors with thin metal (e.g., $\mathrm{Cu}$ and $\mathrm{Al}$ ) layers, which showed a room-temperature responsivity of $0.1-$ $5.5 \mathrm{~mA} / \mathrm{W}$ at the wavelength of $1550 \mathrm{~nm} .^{56,57}$ However, compared to IPE-based Schottky photodetectors using metal silicide layers such as $\mathrm{Pd}_{2} \mathrm{Si}$ and $\mathrm{PtSi}$, which have a maximum responsivity of $50-60 \mathrm{~mA} / \mathrm{W}$ at $1550 \mathrm{~nm}$ at low operating temperatures $(40-140 \mathrm{~K}),^{58-60}$ the $\mathrm{PtSe}_{2}$-based Schottky diodes show inferior performance. Nevertheless, it is expected that the device performance of the $\mathrm{PtSe}_{2}$ photodetectors can be improved by further optimization of fabrication processes. Moreover, the 2D layered feature of $\mathrm{PtSe}_{2}$ is still beneficial for future applications, such as the formation of very thin $2 \mathrm{D}$ heterostructures by stacking the layered $\mathrm{PtSe}_{2}$ with other semiconducting 2D layered TMDs like $\mathrm{MoS}_{2}$ and $\mathrm{WSe}_{2}$.
The thickness dependence of the responsivity of the $\mathrm{PtSe}_{2} / \mathrm{n}$ Si SBDs can be understood by examining the light absorbance of the $\mathrm{PtSe}_{2}$ films: In the energy range above $1.1 \mathrm{eV}$, thinner films absorb less of the incident light than the thicker films, allowing more photons to reach the Si substrate and resulting in a higher responsivity. However, in the IR region where there is no $\mathrm{Si}$ contribution to the responsivity, devices with thicker $\mathrm{PtSe}_{2}$ layers show higher responsivity. This can be attributed to the larger volume for photon absorption in thicker $\mathrm{PtSe}_{2}$ films, allowing more photon-induced charge carriers from $\mathrm{PtSe}_{2}$ to contribute to the photocurrent. This is well supported by the transmittance and absorbance data of $\mathrm{PtSe}_{2}$ films in Figure $4 \mathrm{a}, \mathrm{b}$ (initial Pt thicknesses of 1, 2, 3, and $5 \mathrm{~nm}$, respectively), which was obtained on quartz substrates in the visible to IR wavelength region (400-2000 nm). As expected, thicker films show lower transmittance and higher absorbance than thinner films over the entire wavelength range. In particular, the thinnest $\mathrm{PtSe}_{2}$ film ( $1 \mathrm{~nm}$ starting $\mathrm{Pt}$ ) shows an absorbance of 2 to $15 \%$ in the IR region, which is in the same range of graphene $(\sim 2.3 \%){ }^{61}$ Considering the potential superior manufacturability of $\mathrm{PtSe}_{2}$ due to its low growth temperature and the ease of film-thickness modulation, one can strongly suggest that $\mathrm{PtSe}_{2}$ offers significant advantages over graphene in optoelectronics and has great potential for applications in the IR region.

In summary, we have realized vertical hybrid $\mathrm{PtSe}_{2}$-based diodes and examined their optoelectronic properties. The layered $\mathrm{PtSe}_{2}$ thin films were synthesized at low temperature, with controlled thickness in a scalable and manufacturable manner via TAC of predeposited Pt. The $\mathrm{PtSe}_{2}$ films form a Schottky barrier diode when transferred onto prepatterned n-Si substrates. The SBDs demonstrate a clear photoresponse under illumination and have a comparable responsivity to other 2Dmaterial-based photodiodes. Spectral response measurements reveal that a large part of the photocurrent in the visible wavelength range is generated in the $\mathrm{Si}$ sublayer of the diode. However, in the IR region, $\mathrm{PtSe}_{2}$ acts as a strong absorber with clear thickness dependence. Compared to other IR absorbers based on $2 \mathrm{D}$ materials, like graphene or black phosphorus, $\mathrm{PtSe}_{2}$ is highly advantageous because it can potentially be integrated with standard semiconductor processing due to its low growth temperature. In addition, the absorbance of such devices can be tuned through film thickness modulation. Therefore, this work strongly suggests $\mathrm{PtSe}_{2}$ as a promising 2D material for future IR optoelectronics.

Methods. Layered $\mathrm{PtSe}_{2}$ thin films were synthesized using a TAC process, as detailed in previous work. ${ }^{25,30}$ In short, Pt films of various thicknesses were sputter deposited onto $\mathrm{SiO}_{2} /$ $\mathrm{Si}$ substrates using a Gatan precision etching and coating 
system. These films were converted to $\mathrm{PtSe}_{2}$ in a two-zone furnace with the $\mathrm{Pt}$ films heated to $400{ }^{\circ} \mathrm{C}$ in zone one of the furnace and Se pellets heated to $220{ }^{\circ} \mathrm{C}$ in zone two of the furnace, which was upstream from zone one.

An n-type $\mathrm{Si}\left(\mathrm{n}\right.$-Si) wafer (phosphorus, $5 \times 10^{14} \mathrm{~cm}^{-3}$ $\langle 100\rangle)$ with a thermally grown $\mathrm{SiO}_{2}$ (150 nm thick) layer on top was used to prepare substrates for the devices. A defined area of the $\mathrm{SiO}_{2}$ layer was removed by a hydrofluoric acid (HF, $3 \%$ diluted) wet etching process. After rinsing with deionized (DI) water, metal electrodes (titanium/gold (Ti/Au), 20/50 $\mathrm{nm}$ ) were sputtered on the exposed $\mathrm{n}-\mathrm{Si}$ and the remaining $\mathrm{SiO}_{2}$ region using a shadow mask. After removing the native oxide layer from the $\mathrm{n}$-Si surface using a $\mathrm{HF}$ wet-etching process, as-grown $\mathrm{PtSe}_{2}$ thin films were transferred onto the prepatterned $\mathrm{n}-\mathrm{Si}$ substrates by using a typical polymersupported transfer method with poly(methyl methacrylate) (PMMA, MicroChem). ${ }^{9,25}$ The transferred $\mathrm{PtSe}_{2}$ films are in direct electrical contact with the underlying $\mathrm{n}-\mathrm{Si}$, realizing SBDs with an active area of $\sim 25 \mathrm{~mm}^{2}$. A schematic diagram and photograph of such a device are shown in Figure 2a.

TEM analysis was performed in a JEOL JEM-2100 operating at $200 \mathrm{kV}$ in bright field mode. The lamella was prepared by depositing $50 \mathrm{~nm}$ carbon, $300 \mathrm{~nm}$ platinum, and $2 \mu \mathrm{m}$ carbon protective layer stacks within a dual beam focused ion beam (FIB) FEI Helios NanoLab 600i. The lamella was then thinned down to less than $200 \mathrm{~nm}$ thickness.

Raman spectra of the $\mathrm{PtSe}_{2}$ samples were measured using a Witec Alpha $300 \mathrm{R}$ confocal Raman microscope. The spectra were acquired with an excitation wavelength of $532 \mathrm{~nm}$ and a spectral grating of 1800 lines $/ \mathrm{mm}$.

Hall effect measurements were performed in a van der Pauw configuration with a LakeShore Model 8404 AC/DC Hall Effect Measurement System. The system can provide dc or ac magnetic fields over a variable range up to $\pm 1.7 \mathrm{~T}(\mathrm{dc})$ or a fixed range up to $\sim 1.2 \mathrm{~T}$ RMS at a frequency of $0.1 \mathrm{~Hz}$ (or 0.05 $\mathrm{Hz}$ ).

Electrical characterization of the diodes was conducted under ambient conditions using a Karl Süss probe station connected to a Keithley 2612A source meter unit. The $\mathrm{PtSe}_{2}$ region of the diode was positively biased, and the $\mathrm{n}$-Si was negatively biased. Photoconductivity of the device was tested by using a white light source with a solid state dimmer for variable light intensity (ACE Light Source, SCHOTT A20500, $150 \mathrm{~W}$ halogen lamp).

The spectral response (SR) of the SBDs was measured using a lock-in technique by comparing them to calibrated reference detectors. A monochromator was used to select a particular wavelength of the tungsten-halogen lamp light source. The light power density varied from $1 \mu \mathrm{W} \mathrm{cm} \mathrm{Cm}^{-2}$ at a wavelength of 200 $\mathrm{nm}$ up to $55 \mu \mathrm{W} \mathrm{cm}{ }^{-2}$ at a wavelength of $1150 \mathrm{~nm}$. The intensity of the light beam was modulated by a chopper with a frequency of $17 \mathrm{~Hz}$. Calibrated $\mathrm{Si}$ and indium gallium arsenide (InGaAs) diodes were used for calibration of the setup and also as reference detectors. The photocurrent was measured by preamplifiers and lock-in amplifiers at the working frequency of the chopper for detection of ultralow current values down to 10 pA. A schematic diagram of the SR measurement system is shown in Figure S3 of the Supporting Information.

Transmittance/absorbance data of the $\mathrm{PtSe}_{2}$ films on quartz substrates were measured using a custom-built optical setup. Chopped monochromatic light of a stabilized tungsten-halogen light source and lock-in (EG\&G Model 5210) amplification technique were used to suppress noise and enhance the detection of ultralow current with high accuracy. The optical properties of the films were characterized by using calibrated germanium photodiodes (SN1792), from the visible to the IR range $(3.1-0.6 \mathrm{eV}, 400-2000 \mathrm{~nm})$ with a step size of $0.2 \mathrm{eV}$.

\section{ASSOCIATED CONTENT}

\section{Supporting Information}

The Supporting Information is available free of charge on the ACS Publications website at DOI: 10.1021/acs.nanolett.7b05000.

Details of Hall effect measurements, additional photoresponse data of the $\mathrm{PtSe}_{2} / \mathrm{n}-\mathrm{Si} \mathrm{SBD}$ with various $\mathrm{PtSe}_{2}$ film thicknesses, schematic diagram of the SR measurement system, and additional SR plots of the $\mathrm{PtSe}_{2} / \mathrm{n}-\mathrm{Si}$ SBDs with various $\mathrm{PtSe}_{2}$ film thicknesses (PDF)

\section{AUTHOR INFORMATION}

\section{Corresponding Authors}

*E-mail: lemme@amo.de.

*E-mail: duesberg@unibw.de.

ORCID

Scott Monaghan: 0000-0002-9006-9890

Max C. Lemme: 0000-0003-4552-2411

Notes

The authors declare no competing financial interest.

\section{ACKNOWLEDGMENTS}

Financial support through an ERC grant (InteGraDe, 307311), European Commission grant 696656 (Graphene Flagship), Science Foundation Ireland (15/SIRG/3329 and 15/IA/3131), European Regional Development Funds (HEA2D, EFRE0800149) and the German Research Foundation (DFG LE 2440/1-2, GRK 1564) is gratefully acknowledged.

\section{REFERENCES}

(1) Fiori, G.; Bonaccorso, F.; Iannaccone, G.; Palacios, T.; Neumaier, D.; Seabaugh, A.; Banerjee, S. K.; Colombo, L. Nat. Nanotechnol. 2014, 9 (10), 768-779.

(2) Pospischil, A.; Mueller, T. Appl. Sci. 2016, 6 (3), 78.

(3) Bablich, A.; Kataria, S.; Lemme, M. C. Electronics 2016, 5 (1), 13.

(4) Mak, K. F.; Lee, C.; Hone, J.; Shan, J.; Heinz, T. F. Phys. Rev. Lett. 2010, 105 (13), 136805.

(5) Radisavljevic, B.; Radenovic, A.; Brivio, J.; Giacometti, V.; Kis, A. Nat. Nanotechnol. 2011, 6 (3), 147-150.

(6) Elías, A. L.; Perea-López, N.; Castro-Beltrán, A.; Berkdemir, A.; Lv, R.; Feng, S.; Long, A. D.; Hayashi, T.; Kim, Y. A.; Endo, M.; Gutiérrez, H. R.; Pradhan, N. R.; Balicas, L.; Mallouk, T. E.; LópezUrías, F.; Terrones, H.; Terrones, M. ACS Nano 2013, 7 (6), 52355242.

(7) Allain, A.; Kis, A. ACS Nano 2014, 8 (7), 7180-7185.

(8) Chang, Y.-H.; Zhang, W.; Zhu, Y.; Han, Y.; Pu, J.; Chang, J.-K.; Hsu, W.-T.; Huang, J.-K.; Hsu, C.-L.; Chiu, M.-H.; Takenobu, T.; Li, H.; Wu, C.-I.; Chang, W.-H.; Wee, A. T. S.; Li, L.-J. ACS Nano 2014, 8 (8), 8582-8590.

(9) Yim, C.; O’Brien, M.; McEvoy, N.; Riazimehr, S.; SchaferEberwein, H.; Bablich, A.; Pawar, R.; Iannaccone, G.; Downing, C.; Fiori, G.; Lemme, M. C.; Duesberg, G. S. Sci. Rep. 2015, 4, 5458.

(10) Lee, Y.-H.; Zhang, X.-Q.; Zhang, W.; Chang, M.-T.; Lin, C.-T.; Chang, K.-D.; Yu, Y.-C.; Wang, J. T.-W.; Chang, C.-S.; Li, L.-J.; Lin, T.W. Adv. Mater. 2012, 24 (17), 2320-2325.

(11) Zhan, Y.; Liu, Z.; Najmaei, S.; Ajayan, P. M.; Lou, J. Small 2012, 8 (7), 966-971.

(12) Lu, X.; Utama, M. I. B.; Lin, J.; Gong, X.; Zhang, J.; Zhao, Y.; Pantelides, S. T.; Wang, J.; Dong, Z.; Liu, Z.; Zhou, W.; Xiong, Q. Nano Lett. 2014, 14 (5), 2419-2425. 
(13) Gatensby, R.; McEvoy, N.; Lee, K.; Hallam, T.; Berner, N. C.; Rezvani, E.; Winters, S.; O’Brien, M.; Duesberg, G. S. Appl. Surf. Sci. 2014, 297, 139-146.

(14) Liu, H. F.; Wong, S. L.; Chi, D. Z. Chem. Vap. Deposition 2015, 21, 241-259.

(15) Bosi, M. RSC Adv. 2015, 5 (92), 75500-75518.

(16) Li, J.; Östling, M. Electronics 2015, 4 (4), 1033-1061.

(17) Lee, K.; Gatensby, R.; McEvoy, N.; Hallam, T.; Duesberg, G. S. Adv. Mater. 2013, 25 (46), 6699-6702.

(18) Luo, S.; Qi, X.; Ren, L.; Hao, G.; Fan, Y.; Liu, Y.; Han, W.; Zang, C.; Li, J.; Zhong, J. J. Appl. Phys. 2014, 116 (16), 164304.

(19) Zhang, W.; Chuu, C.-P.; Huang, J.-K.; Chen, C.-H.; Tsai, M.-L.; Chang, Y.-H.; Liang, C.-T.; Chen, Y.-Z.; Chueh, Y.-L.; He, J.-H.; Chou, M.-Y.; Li, L.-J. Sci. Rep. 2015, 4, 3826.

(20) Kang, K.; Xie, S.; Huang, L.; Han, Y.; Huang, P. Y.; Mak, K. F.; Kim, C.-J.; Muller, D.; Park, J. Nature 2015, 520 (7549), 656-660.

(21) Campbell, P. M.; Tarasov, A.; Joiner, C. A.; Tsai, M.-Y.; Pavlidis, G.; Graham, S.; Ready, W. J.; Vogel, E. M. Nanoscale 2016, 8 (4), $2268-2276$

(22) Wilson, J. A.; Yoffe, A. D. Adv. Phys. 1969, 18 (73), 193-335.

(23) Guo, G. Y.; Liang, W. Y. J. Phys. C: Solid State Phys. 1986, 19 (7), 995.

(24) Miró, P.; Ghorbani-Asl, M.; Heine, T. Angew. Chem., Int. Ed. 2014, 53 (11), 3015-3018.

(25) Yim, C.; Lee, K.; McEvoy, N.; O’Brien, M.; Riazimehr, S.; Berner, N. C.; Cullen, C. P.; Kotakoski, J.; Meyer, J. C.; Lemme, M. C.; Duesberg, G. S. ACS Nano 2016, 10 (10), 9550-9558.

(26) Zhao, Y.; Qiao, J.; Yu, P.; Hu, Z.; Lin, Z.; Lau, S. P.; Liu, Z.; Ji, W.; Chai, Y. Adv. Mater. 2016, 28 (12), 2399-2407.

(27) Wang, Z.; Li, Q.; Besenbacher, F.; Dong, M. Adv. Mater. 2016, 28 (46), 10224-10229.

(28) Zhao, Y.; Qiao, J.; Yu, Z.; Yu, P.; Xu, K.; Lau, S. P.; Zhou, W.; Liu, Z.; Wang, X.; Ji, W.; Chai, Y. Adv. Mater. 2017, 29 (5), 1604230. (29) Zhuang, H. L.; Hennig, R. G. J. Phys. Chem. C 2013, 117 (40), 20440-20445

(30) O’Brien, M.; McEvoy, N.; Motta, C.; Zheng, J.-Y.; Berner, N. C.; Kotakoski, J.; Elibol, K.; Pennycook, T. J.; Meyer, J. C.; Yim, C.; Abid, M.; Hallam, T.; Donegan, J. F.; Sanvito, S.; Duesberg, G. S. $2 D$ Mater. 2016, 3 (2), 021004.

(31) Wang, Y.; Li, L.; Yao, W.; Song, S.; Sun, J.; Pan, J.; Ren, X.; Li, C.; Okunishi, E.; Wang, Y.-Q.; Wang, E.; Shao, Y.; Zhang, Y.; Yang, H.; Schwier, E. F.; Iwasawa, H.; Shimada, K.; Taniguchi, M.; Cheng, Z.; Zhou, S.; Du, S.; Pennycook, S. J.; Pantelides, S. T.; Gao, H.-J. Nano Lett. 2015, 15 (6), 4013-4018.

(32) Wang, Q.; Lai, J.; Sun, D. Opt. Mater. Express 2016, 6 (7), 2313.

(33) Kim, J.; Li, S. S.; Figueroa, L.; Carruthers, T. F.; Wagner, R. S. J. Appl. Phys. 1988, 64 (11), 6536-6540.

(34) Tzeng, Y. C.; Li, S. S.; Ho, P. IEEE Trans. Electron Devices 1993, 40 (2), 348-352.

(35) Wohlmuth, W. A.; Seo, J. W.; Fay, P.; Caneau, C.; Adesida, I.

IEEE Photonics Technol. Lett. 1997, 9 (10), 1388-1390.

(36) Biyikli, N.; Kimukin, I.; Butun, B.; Aytur, O.; Ozbay, E. IEEE J. Sel. Top. Quantum Electron. 2004, 10 (4), 759-765.

(37) An, X.; Liu, F.; Jung, Y. J.; Kar, S. Nano Lett. 2013, 13 (3), 909916.

(38) Riazimehr, S.; Bablich, A.; Schneider, D.; Kataria, S.; Passi, V.; Yim, C.; Duesberg, G. S.; Lemme, M. C. Solid-State Electron. 2016, 115, 207-212.

(39) Yim, C.; Passi, V.; Lemme, M. C.; Duesberg, G. S.; Coileáin, C. Ó.; Pallechi, E.; Fadil, D.; McEvoy, N. arXiv:1707.06824 [cond-mat], 2017.

(40) Ali Umar, A.; Md Saad, S. K.; Mat Salleh, M. ACS Omega 2017,

2 (7), 3325-3332.

(41) Sedky, S.; Witvrouw, A.; Bender, H.; Baert, K. IEEE Trans. Electron Devices 2001, 48 (2), 377-385.

(42) Takeuchi, H.; Wung, A.; Sun, X.; Howe, R. T.; King, T.-J. IEEE

Trans. Electron Devices 2005, 52 (9), 2081-2086.

(43) Lee, Y. H. D.; Lipson, M. IEEE J. Sel. Top. Quantum Electron. 2013, 19 (2), 8200207.
(44) Nazir, G.; Khan, M. F.; Iermolenko, V. M.; Eom, J. RSC Adv. 2016, 6 (65), 60787-60793.

(45) Pradhan, N. R.; Rhodes, D.; Memaran, S.; Poumirol, J. M.; Smirnov, D.; Talapatra, S.; Feng, S.; Perea-Lopez, N.; Elias, A. L.; Terrones, M.; Ajayan, P. M.; Balicas, L. Sci. Rep. 2015, 5, 8979.

(46) Rhoderick, E. H. IEE Proc., Part I: Solid-State Electron Devices 1982, 129 (1), 1-14.

(47) Sze, S. M.; Ng, K. K. Physics of Semiconductor Devices, 3rd ed.; John Wiley \& Sons, Inc.: Hoboken, NJ, 2007.

(48) Cheung, S. K.; Cheung, N. W. Appl. Phys. Lett. 1986, 49 (2), $85-87$.

(49) Li, X.; Zhu, M.; Du, M.; Lv, Z.; Zhang, L.; Li, Y.; Yang, Y.; Yang, T.; Li, X.; Wang, K.; Zhu, H.; Fang, Y. Small 2016, 12 (5), 595-601.

(50) Choi, W.; Cho, M. Y.; Konar, A.; Lee, J. H.; Cha, G.-B.; Hong, S.

C.; Kim, S.; Kim, J.; Jena, D.; Joo, J.; Kim, S. Adv. Mater. 2012, 24 (43), 5832-5836.

(51) Esmaeili-Rad, M. R.; Salahuddin, S. Sci. Rep. 2013, 3, 2345.

(52) Casalino, M. IEEE J. Quantum Electron. 2016, 52 (4), 1-10.

(53) Gatensby, R.; Hallam, T.; Lee, K.; McEvoy, N.; Duesberg, G. S. Solid-State Electron. 2016, 125, 39-51.

(54) Peters, D. W. Proc. IEEE 1967, 55 (5), 704-705.

(55) Flohr, T.; Schulz, M. Appl. Phys. Lett. 1986, 48 (22), 15341535.

(56) Lee, M.-K.; Chu, C.-H.; Wang, Y.-H.; Sze, S. M. Opt. Lett. 2001, 26 (3), 160-162.

(57) Casalino, M.; Sirleto, L.; Iodice, M.; Saffioti, N.; Gioffrè, M.; Rendina, I.; Coppola, G. Appl. Phys. Lett. 2010, 96 (24), 241112.

(58) Elabd, H.; Villani, T.; Kosonocky, W. IEEE Electron Device Lett. 1982, 3 (4), 89-90.

(59) Tsaur, B. Y.; Weeks, M. M.; Pellegrini, P. W. IEEE Electron Device Lett. 1988, 9 (2), 100-102.

(60) Chen, C. K.; Nechay, B.; Tsaur, B. Y. IEEE Trans. Electron Devices 1991, 38 (5), 1094-1103.

(61) Nair, R. R.; Blake, P.; Grigorenko, A. N.; Novoselov, K. S.; Booth, T. J.; Stauber, T.; Peres, N. M. R.; Geim, A. K. Science 2008, 320 (5881), 1308-1308. 